\title{
Burden of Care as Perceived by Informal Caregivers of the Patients Receiving Chemotherapy in Tertiary Care Hospital of Karachi, Pakistan
}

\author{
Asher Ghori', Rubina Barolia², Naghma Rizvi², Ghulam Qadir3 \\ ${ }^{1}$ Male School of Nursing, Sindh Government Hospital Liaquatabad, Karachi, Pakistan \\ ${ }^{2}$ School of Nursing and Midwifery, Aga Khan University, Karachi, Pakistan \\ ${ }^{3}$ Sindh Government Hospital, Karachi, Pakistan \\ Email: asherghori@gmail.com, rubina.barolia@aku.edu,naghma.rizvi@aku.edu,balochqadir1987@gmail.com
}

How to cite this paper: Ghori, A., Barolia, R., Rizvi, N. and Qadir, G. (2020) Burden of Care as Perceived by Informal Caregivers of the Patients Receiving Chemotherapy in Tertiary Care Hospital of Karachi, Pakistan. Open Journal of Emergency Medicine, 8, 95-109.

https://doi.org/10.4236/ojem.2020.84011

Received: November 9, 2019

Accepted: December 13, 2020

Published: December 16, 2020

Copyright $\odot 2020$ by author(s) and Scientific Research Publishing Inc. This work is licensed under the Creative Commons Attribution International License (CC BY 4.0).

http://creativecommons.org/licenses/by/4.0/

\begin{abstract}
Background: Almost 32.5 million people are diagnosed with cancer annually. The huge number of cancer patients with deteriorating health renders a far greater number of informal caregivers bearing the burden of care in different forms and magnitude. The main purpose of the study was to explore the types of burden perceived by the informal caregivers and the factors associated with the caregiving burden. Methods. A quantitative descriptive cross-sectional study design was adopted to answer the research questions from April to May 2016. Around 200 informal caregivers were recruited from one of the tertiary hospitals of Karachi, Pakistan. The modified ZBIS was used to collect data which was analyzed by using Chi-square test to find the associations between the demographic characteristics of informal caregivers and the perceived burden of care. Results. Findings of the study identified the employment status $(P \leq 0.020)$ and the religion of the informal caregivers $(P \leq 0.027)$ as significant, demographic variables. The functional level of care receivers was also found to be significant $(\mathrm{P} \leq 0.002)$. The study also recognized four forms of burdens, with significant mean values, including financial (2.85), psychological (2.50), social (2.58), and physical (2.44). Most of the participants reported experiencing a moderate magnitude of burden, ranging from 40 - 60, on the scale of 0 - 80. Conclusion: The study highlighted four different forms of burdens, that is, financial, psychological, social, and physical, along with their associated demographic factors. Health care agencies and public health
\end{abstract}


personnel should work with informal caregivers to reduce burden of care.

\section{Keywords}

Burden of Care, Informal Caregiver, Chemotherapy

\section{Introduction}

Globally, cancer accounts for 7.6 million deaths per year and the death rate is expected to increase up to 13.5 million in 2030 [1]. Chemotherapy has rapidly become a major present day modality for cancer treatment [2]. However, along with its healing effects, chemotherapy also produces certain adverse effects which include alopecia, nausea, vomiting, anorexia, sudden weight loss [3], depression, anxiety, and cancer-related fatigue [4]. Another common side effect of chemotherapy includes cognitive deficit which entails concentration impairment, attention deficit, decreased self-confidence and patients requiring more time and effort to complete regular tasks [5]. Thus, these cancer patients become heavily dependent upon health care personnel and family members (informal caregivers) to manage daily activities [6].

The informal caregiver is defined as an unpaid care provider who suffers physically, psychologically, emotionally, and socially from the burden of care [7] and, as such, is tasked with assisting the cancer patient with basic daily activities, providing psychological support and prompt access to healthcare facilities [8]. However, it was found that caregivers were not fully prepared and trained to meet the challenges that arise during the initial caregiving period [9]. Studies show that informal caregivers develop negative coping mechanisms, which include but are not limited to: alcohol consumption, smoking and immobility [10]. Additionally, the more social roles the caregivers have to play the more is the stress and negative impact on their health i.e. significant levels of depression, anxiety, sleep dysfunction, and increased blood pressure [11] [12]. The care giving experience alters the caregiver's relationship with other family members and friends and disturbs the psychological, occupational, financial, and physical domains of the care giver.

This leads to the conclusion that the debilitating burden of responsibilities on the informal caregiver decreases the quality of care provided to patients, ultimately affecting their health [13] [14]. Hence, it is essential to reduce the burden of care on caregivers such that their and the care-receiver's health does not suffer, and to further bolster the quality of care provided. To date, no study from Pakistan has been carried out that investigated cancer-related mortality or the burdens associated with taking care of a cancer patient. Therefore, this study was carried out with the specific intent of improving the quality of care provided to patients receiving chemotherapy by assessing the perceived burden of care by informal caregivers and to make recommendations that would lessen it. 


\section{Methods}

\subsection{Study Design, Setting, and Study Population}

A quantitative, descriptive cross-sectional study design was adopted to answer the research questions of this study. The target population comprised of informal caregivers who were the friends or blood relatives of patients and had been providing care without any monetary compensation. The sample consisted of a convenient sampling of 200 recruited from the day-care chemotherapy unit of the Aga Khan University Hospital (AKUH) in Karachi, Pakistan from April to May 2016.

\subsection{Sampling, and Eligibility Criteria}

The sample size was calculated using the Open Epi Software version 3.03 and the prevalence of the informal caregiving burden, which was estimated to be 377 , however, due to time constraints, it was reduced to 200. Caregivers of both genders were eligible to participate in the study on the basis that 1) they could speak English or Urdu, 2) were aged 18 years or older, 3) had been providing care to the cancer patient receiving chemotherapy for at least six months. Informal caregivers providing care to the cancer patients receiving treatment modality other than chemotherapy or who refused to participate in the study were excluded. The level of dependency of cancer patients on their informal caregivers was found to be mild to moderate only.

\subsection{Procedure and Study Tool}

The study was approved by the Ethical Review Committee of AKUH, Karachi and permission for data collection was taken from the head of chemotherapy department, AKUH. The head nurse of the oncology ward taken on board, because she could access the private and confidential files of the oncology patients and had knowledge about the chemotherapy plan, facilitated the selection of the study participants. Verbal and informed written consent was obtained from the participants before data collection.

Demographical data was collected by a self-generated questionnaire which also determined the socio-demographical characteristics of the informal caregivers. The questionnaire consisted of 14 questions which provided information about the gender, age, employment, religion and functional status of the care receivers. Approximately 15 - 20 minutes were required to fill each questionnaire. The Modified Zarit Burden Interview Scale (MZBIS) was utilized to identify the burden of care as perceived by the informal caregiver of the patient receiving chemotherapy. This tool is based on the Likert scale and was selected because it covers all the aspects of caregiving that impose a burden on the informal caregiver, including the physical, psychological, social, and financial burdens. As the ZBIS scale has been used by different researchers in various countries and settings, some of the variables in the tool were modified according to the Pakistani context (Table 1) [15]. 
Table 1. Content validity index.

\begin{tabular}{cc}
\hline Content Validity & Scores \\
\hline Relevancy & 0.96 \\
Clarity & 0.94 \\
\hline
\end{tabular}

The Modified Zarit Burden Interview Scale (MZBIS) was utilized to identify the burden of care as perceived by the informal caregiver of the patient receiving chemotherapy. Internal validity of the study was maintained by carrying out a pilot study on $10 \%$ of the participants. Pre-testing was used to ensure that the questionnaire was easily comprehended by the participants. Content validity was ensured by sending the English and Urdu versions of ZBIS to two oncologists, the nurse manager of the oncology unit, and one of the experienced nurses of the oncology unit for their feedback and the necessary amendments were made accordingly.

Initially, individuals who were willing to participate in the study voluntarily were identified by the head nurse and verbal consent was obtained from them. In the second step, the researcher approached the identified caregivers and obtained the written consent from them, before starting data collection. In order to maintain the privacy of the participants' counseling room of the oncology day care was used for interview purposes.

\subsection{Statistical Analysis}

The data was collected by the principal investigator (PI) and entered on a daily basis to ensure comprehensiveness and consistency in recording. The statistical software Statistical Package for Social Sciences SPSS version 19 was picked for the purpose of data entry. For categorical variables, frequencies and proportions were utilized whereas, for the continuous variables, descriptive statistics were utilized to compute mean and standard deviation. Chi square analysis was used to find out the factors associated with the burden of the caregiver.

\section{Results}

The caregivers were asked to mark the burden of care on a Likert scale, which ranged from $1-4$, representing strongly disagree to strongly agree, respectively. The mean $( \pm \mathrm{SD})$ score for overall burden was computed and found to be 2.67 $( \pm 0.514)$, indicating that all caregivers recognized the burden of care. Each question in MZBIS had four possible responses that were: strongly disagree, disagree, agree, and strongly agree. Responses were scored from 1 to 4 and the total scores were calculated by obtaining the sum of scores of all the questions. Final scores ranged from 0 to 80 , of which 0 - 20 referred to no burden, 21 - 40 showed mild burden, 41 - 60 referred to moderate burden, and 60 - 80 showed severe burden. The magnitude of the burden was computed according to the scale mentioned above and it was found that almost $89 \%(\mathrm{n}=178)$ of the participants experienced moderate burden of care (Table 2). 
Table 2. Forms of burden with prevalence.

\begin{tabular}{ccc}
\hline Forms of burden & Mean & \pm SD \\
\hline Financial burden & 2.85 & \pm 0.550 \\
Social burden & 2.58 & \pm 0.457 \\
Psychological burden & 2.50 & \pm 0.370 \\
Physical burden & 2.44 & \pm 0.343 \\
\hline
\end{tabular}

\subsection{Forms of Burden}

The mean $( \pm S D)$ score for each form of perceived burden, computed individually on a four point Likert scale, showed financial burden $2.8( \pm 0.550)$ as the highest, followed by psychological burden $2.5( \pm 0.370)$, and social burden 2.5.

\section{Basic Socio-Demographic Characteristics of the Informal Caregivers}

A total of 200 informal caregivers were approached in order to explore the burden of care observed, along with its forms and perceived magnitude. The data was collected between the months of April and May, 2016. Half of the participants 52.5\% ( $n=105)$ were males, and $40 \%(n=80)$ fell between 20 - 34 years of age. The data revealed that $66 \%(n=132)$ of the participants were married, while the religious background of the caregivers depicted that around $93.5 \%(\mathrm{n}=187)$ were Muslims (as shown in Table 3).

\section{Socio-demographic Characteristics of Informal Caregivers}

The data revealed that $47.5 \%(\mathrm{n}=95)$ were employed and $52.5 \%(\mathrm{n}=105)$ were non-employed; among the non-employed, most of the participants were house wives. Results for the academic qualification showed that $32 \%(n=64)$ of the participants had a bachelor's degree and $26.0 \%(n=52)$ had a master's degree. As far as the monthly income of the caregiver was concerned, it was found that $33 \%(n=67)$ of the care givers were generating a monthly revenue of Rs. 5000 to 34,000 , and around $32 \%(n=64)$ were earning Rs.35,000 to 64,000 (as shown in Table 4).

\section{Relationship, Comorbidity and Total Number of Caregivers Available}

An analysis of the range of relationship of the caregivers revealed that $26 \%$ ( $\mathrm{n}$ $=52)$ of the participants were daughters, $26 \%(n=52)$ siblings, and $21 \%(n=43)$ spouses. Almost half $44.5 \%(\mathrm{n}=89)$ of the care receivers had two to three informal care givers at home, whereas $37 \%(\mathrm{n}=74)$ had more than three caregivers. The majority of the caregivers $(71 \%, \mathrm{n}=142)$ did not have any comorbidity (shown in Table 5).

\subsection{Demographical Characteristics of Care Receivers}

The functional level of the care receivers showed that the majority $86.5 \%(\mathrm{n}=$ 173) were independent (Table 6).

\subsection{Significant Demographic Variables}

The chi-square test was applied to each socio-demographic variable of caregivers 
to assess their association with the burden perceived. Results revealed that three variables, including employment status of the caregivers $(P \leq 0.020)$, functional level of the care receivers $(P \leq 0.002)$, and religion $(P \leq 0.027)$, were statistically significant.

Table 3. Demographic characteristics of the informal caregivers at AKUH Karachi $(\mathrm{n}=$ 200).

\begin{tabular}{ccc}
\hline Characteristics & $\mathrm{n}$ & $(\%)$ \\
\hline Age (in years) & & \\
20 years - 34 years & 80 & $(40.0)$ \\
35 years - 49 years & 65 & $(32.5)$ \\
50 years and above & 55 & \\
Gender & 105 & \\
Male & 95 & $(52.5)$ \\
Female & & \\
Marital Status & 64 & $(32.0)$ \\
Single & 132 & $(66.0)$ \\
Married & 4 & $(2.0)$ \\
Widow/Widower & & \\
Religion & 187 & $(93.5)$ \\
Islam & 3 & $(1.5)$ \\
Christianity & 10 & \\
Hinduism & &
\end{tabular}

Table 4. Employment, education, and income status of the informal caregivers.

\begin{tabular}{ccc}
\hline Characteristics & $\mathbf{n}$ & $(\%)$ \\
\hline Employment Status & 105 & $(52.5)$ \\
$\quad$ Not Employed & 95 & $(47.5)$ \\
Employed & & \\
Academic Qualification of caregivers & 28 & $(14.0)$ \\
Under-Matriculate & 27 & $(13.5)$ \\
Matriculate/ "O" level & 26 & $(13.0)$ \\
Intermediate/ "A" Level & 64 & $(32.0)$ \\
Bachelors & 52 & $(26.0)$ \\
Masters & 3 & $(1.5)$ \\
PhD & & \\
Income of the Caregiver per month & 67 & $(33.5)$ \\
5000 - 34,000 & 64 & $(32.0)$ \\
35,000 - 64,000 & 18 & $(9.0)$ \\
65,000 - 94,000 & 51 & $(25.5)$ \\
95,000 and more &
\end{tabular}


Table 5. Descriptive features of informal caregivers.

\begin{tabular}{ccc}
\hline Characteristics & $\mathbf{n}$ & $(\%)$ \\
\hline Relationship to Patient & & $(21.5)$ \\
Spouse & 43 & $(18.0)$ \\
Son & 36 & $(26.0)$ \\
Daughter & 52 & $(3.5)$ \\
Parent & 7 & $(26.0)$ \\
Sibling & 52 & $(5.0)$ \\
Friend & 10 & \\
Total Number of Caregivers & & $(18.5)$ \\
0 - 1 & 37 & $(44.5)$ \\
2 - 3 & 89 & $(37)$ \\
More than 3 & 74 & $(71 \%)$ \\
Co-morbidity & & $(29 \%)$ \\
No Co-morbidity & 142 & \\
Co-morbidity & 58 & \\
\hline
\end{tabular}

Table 6. Descriptive characteristics of the care receivers.

\begin{tabular}{|c|c|c|}
\hline Characteristics & $\mathrm{N}$ & $(\%)$ \\
\hline \multicolumn{3}{|l|}{ Type of Cancer } \\
\hline Breast cancer & 69 & $(34.5)$ \\
\hline Ovarian & 30 & $(15.0)$ \\
\hline Osteosarcoma & 26 & $(13.0)$ \\
\hline Lymphoma & 16 & $(8.0)$ \\
\hline Colon & 3 & $(1.5)$ \\
\hline Other types of cancer & 56 & $(28.0)$ \\
\hline \multicolumn{3}{|l|}{ Grading of Cancer } \\
\hline Stage 1 & 16 & $(8.0)$ \\
\hline Stage 2 & 52 & $(26.0)$ \\
\hline Stage 3 & 57 & $(28.5)$ \\
\hline Metastasis & 75 & $(37.5)$ \\
\hline \multicolumn{3}{|c|}{ Total number of months of receiving chemotherapy } \\
\hline 6 to 11 months & 94 & $(47)$ \\
\hline 12 to 17 months & 38 & (19) \\
\hline 18 to 23 months & 8 & (4) \\
\hline 24 to 29 months & 20 & $(10)$ \\
\hline 30 or more months & 40 & $(20)$ \\
\hline \multicolumn{3}{|c|}{ Functional level of care receivers } \\
\hline Independent & 173 & $(86.5)$ \\
\hline Required assistance & 24 & $(12)$ \\
\hline Completely dependent & 3 & $(1.5)$ \\
\hline
\end{tabular}




\subsection{Perceived Physical and Psychological Burden}

The perceived physical burden was significantly higher during the care giving duration, as $59.5 \%(\mathrm{n}=119)$ informal caregivers stated that their care receivers were physically dependent upon them and a majority $(n=165)$, acknowledged that better functional level of the care receivers played an important role in reducing the burden of care. Participants also reported that the care giving burden disrupted their relationship with family and friends.

Results also revealed that the psychological burden affects both the care receivers and the informal caregivers equally. Almost 57.5\% $(n=115)$ of the caregivers reported to be frustrated over the inconsistent behavior of the care receivers. Participants also expressed their discomfort over their care receivers' irritable behavior; whereas, $76 \%(n=152)$ were found to be afraid of the imminent negative consequences of the disease.

\subsection{Participants' Perception about Professional Support}

All the caregivers endorsed the need for professional support in unison and agreed that it is important to reduce the burden of care.

\subsection{Basic Socio-Demographic Characteristics of the Informal Caregivers}

A total of 200 informal caregivers were approached in order to explore the burden of care observed, along with its forms and perceived magnitude. Half of the participants $52.5 \%(\mathrm{n}=105)$ were males, and $40 \%(\mathrm{n}=80)$ fell between $20-34$ years of age. The data revealed that $66 \%(n=132)$ of the participants were married, whereas around $93.5 \%(n=187)$ were Muslims.

\section{Perceived Magnitude of Burden}

The caregivers were asked to mark the burden of care on a Likert scale, which ranged from $1-4$, representing strongly disagree to strongly agree, respectively (refer to appendix). The mean $( \pm \mathrm{SD})$ score for overall burden was computed and found to be 2.67 ( \pm 0.514 ), indicating that all caregivers recognized the burden of care. Each question in ZBIS had four possible responses that were: strongly disagree, disagree, agree, and strongly agree. Responses were scored from 1 to 4 and the total scores were calculated by obtaining the sum of scores of all the questions. Final scores ranged from 0 to 80 , of which 0 - 20 referred to no burden, 21 - 40 showed mild burden, 41 - 60 referred to moderate burden, and 60 - 80 showed severe burden. The magnitude of the burden was computed according to the scale mentioned above and it was found that almost $89 \%(\mathrm{n}=178)$ of the participants experienced moderate burden of care (as shown in Table 7).

\section{Inferential Statistics}

\section{Demographic Characteristics and Perceived Burden}

The chi-square statistics test was applied on all the demographic variables to discover significant association with the dependent variable, i.e., Burden of care. The results revealed that some of the demographical variables were found to be statistically significant (as shown in Table 8). 
Table 7. Magnitude of burden.

\begin{tabular}{ccc}
\hline Magnitude & n (\%) & P-values \\
\hline Mild burden & $9(4.5)$ & \\
Moderate burden & $178(89)$ & $0.001^{*}$ \\
Severe burden & $13(6.5)$ & \\
\hline
\end{tabular}

*Significant $\mathrm{p}$-values of chi-square test $(\mathrm{P} \leq 0.05)$.

Table 8. Analyses of demographic characteristics with perceived burden of care.

\begin{tabular}{cc}
\hline Variables & $P$-values \\
\hline Gender & 0.776 \\
Age & 0.536 \\
Employment & $0.020^{*}$ \\
Religion & $0.027^{*}$ \\
Relation to caregiver & 0.987 \\
Total number of caregivers & 0.317 \\
Marital status & 0.612 \\
Academic qualification & 0.748 \\
Co-morbidity & 0.068 \\
Total number of months of receiving chemotherapy & 0.608 \\
Type of cancer & 0.517 \\
Staging of cancer & 0.147 \\
Functional level of care receiver & $0.002^{*}$ \\
Income of care giver & 0.697 \\
\hline
\end{tabular}

${ }^{*} P$-value $(\mathrm{P} \leq 0.05)$ of Chi-square test.

\section{Significant Demographic Variables}

The chi-square test was applied to each socio-demographic variable of caregivers to assess their association with the burden perceived. Results revealed that three variables, including employment status of the caregivers, functional level of the care receivers, and religion, were statistically significant (as shown in Tables 9-11).

\section{Analysis of the Modified Zarit Burden Interview Scale}

Similarly, each variable from each domain underwent the chi-square statistic and the results revealed that each form of the burden emerged with statistically significant weightage. Regarding the financial domain, 59.5\% $(n=119)$ of the participants reported that their care receivers were dependent upon the caregivers, and almost $83 \%(n=166)$ of the participants were facing financial issues, while $51 \%(n=102)$ agreed that the care receivers were exerting more financial burden than what could be tolerated. The descriptive statistics of the four domains financial burden, physical burden, psychological burden, and social burden that were found to be significant are shown in Table 12 . 
Table 9. Employment status.

\begin{tabular}{ccc}
\hline & $\mathbf{n}(\%)$ & P-values \\
\hline Not Employed & $105(52.5)$ & $0.020^{*}$ \\
Employed & $95(47.5)$ & \\
\hline
\end{tabular}

"Significant p-values of chi-square test $(\mathrm{P} \leq 0.05)$.

Table 10. Functional levels of care receiver and burden perceived.

\begin{tabular}{ccc}
\hline & $\mathbf{n}(\%)$ & P-value \\
\hline Independent & $173(86.5)$ & \\
Required assistance & $24(12)$ & $0.002^{*}$ \\
Completely Dependent & $3(1.5)$ & \\
\hline
\end{tabular}

* Significant p-values of chi-square test $(\mathrm{P} \leq 0.05)$.

Table 11. Religions of caregivers and burden perceived.

\begin{tabular}{ccc}
\hline & $\mathbf{n}(\%)$ & P-values \\
\hline Islam & $187(93.5)$ & \\
Hinduism & $10(5)$ & $0.027^{*}$ \\
Christianity & $3(1.6)$ & \\
\hline
\end{tabular}

* Significant $\mathrm{p}$-values of chi-square test $(\mathrm{P} \leq 0.05)$.

Table 12. Perceived financial burden.

\begin{tabular}{cccc}
\hline Question Statement & No Burden & Burden & p-value \\
\hline $\begin{array}{c}\text { Do you feel that your relative is } \\
\text { dependent upon you? }\end{array}$ & $81(40.5 \%)$ & $119(59.5 \%)$ & 0.015 \\
$\begin{array}{c}\text { Do you feel that you face financial issues } \\
\text { while taking care of your relative, in addition } \\
\text { to the rest of your expenses? }\end{array}$ & $34(17 \%)$ & $166(83 \%)$ & 0.000 \\
$\begin{array}{c}\text { Do you feel that your relative is expecting } \\
\text { more from you as you are the only person } \\
\text { he/she can depend upon? }\end{array}$ & $98(49 \%)$ & $102(51 \%)$ & 0.002 \\
\hline
\end{tabular}

${ }^{*} \mathrm{p}$-values $(\mathrm{P} \leq 0.05)$.

The perceived physical burden was significantly higher during the care giving duration, as $59.5 \%(n=119)$ informal caregivers stated that their care receivers were physically dependent upon them and a majority, $82.5 \%(\mathrm{n}=165)$, acknowledged the fact that better functional level of the care receivers plays an important role in reducing the burden of care (as shown in Table 13).

The results also revealed that the psychological burden affects both the care receivers and the informal caregivers equally. Almost 57.5\% $(n=115)$ of the caregivers reported to be frustrated over the inconsistent behavior of the care receivers. Around $69 \%(\mathrm{n}=138)$ of the participants expressed their discomfort over their care receivers' irritable behavior; whereas, $76 \%(n=152)$ were found to be afraid of the imminent negative consequences of the disease (as shown in 


\section{Table 14).}

The social burden affects the informal care givers in a negative way, as 50.5\% ( $\mathrm{n}=101)$ of the caregivers reported that their relationship with other family members was disrupted (as shown in Table 15).

Participants' Perception about Professional Support

All the caregivers endorsed the need for professional support in unison and agreed that it is important to reduce the burden of care (shown in Tables 16-18).

Table 13. Perceived physical burden.

\begin{tabular}{cccc}
\hline Question Statement & No Burden & Burden & P-Value \\
\hline $\begin{array}{c}\text { Do you feel that your } \\
\text { relative is dependent upon you? }\end{array}$ & $81(40 \%)$ & $119(59.5 \%)$ & 0.015 \\
$\begin{array}{c}\text { Do you feel that a better functional level of } \\
\text { your relative may lessen the burden of care? }\end{array}$ & $35(17.5 \%)$ & $165(82.5 \%)$ & 0.003 \\
$\begin{array}{c}\text { Do you feel that your health is getting adversely } \\
\text { affected while taking care of your relative? }\end{array}$ & $108(54 \%)$ & $92(46 \%)$ & 0.000 \\
\hline
\end{tabular}

${ }^{*} \mathrm{p}$-values $(\mathrm{P} \leq 0.05)$.

Table 14. Perceived psychological burden.

\begin{tabular}{|c|c|c|c|}
\hline Question Statement & No Burden & Burden & $P$-value \\
\hline Do you feel frustrated over your relative's behavior? & $85(42.5 \%)$ & $115(57.5 \%)$ & 0.001 \\
\hline $\begin{array}{l}\text { Do you feel that your relative is } \\
\text { irritable and not willing to receive care? }\end{array}$ & $62(31 \%)$ & $138(69 \%)$ & 0.000 \\
\hline $\begin{array}{l}\text { Are you afraid of what the } \\
\text { future holds for your relative? }\end{array}$ & $48(24 \%)$ & $152(76 \%)$ & 0.011 \\
\hline
\end{tabular}

${ }^{*}$-values $(\mathrm{P} \leq 0.05)$.

Table 15. Perceived social burden.

\begin{tabular}{cccc}
\hline Question Statement & No Burden & Burden & P-value \\
\hline $\begin{array}{c}\text { Do you feel that caregiving currently } \\
\text { adversely affects your relationship } \\
\text { with other family members and friends? } \\
\begin{array}{c}\text { Do you feel that your privacy is } \\
\text { compromised during the caregiving period? }\end{array}\end{array}$ 158 (79\%) & $42(21 \%)$ & 0.001 \\
\hline
\end{tabular}

${ }^{*} \mathrm{p}$-values $(\mathrm{P} \leq 0.05)$.

Table 16. Chi-square test statistic of Q.21.

Do you think that professionals, help can enhance your caregiving skill?

\begin{tabular}{ccc}
\hline & $\mathbf{n}(\%)$ & P-values \\
Strongly Disagree & $2(1)$ & \\
Disagree & $19(9.5)$ & $0.000^{* *}$ \\
Agree & $82(41)$ & \\
Strongly agree & $97(48.5)$ & \\
\hline
\end{tabular}

"* Significant p-values of chi-square test $(\mathrm{P} \leq 0.05)$. 
Table 17. Chi-square test statistic of Q.22.

\begin{tabular}{ccc}
\multicolumn{2}{c}{ Do you think that observing professionals while } \\
performing the skills is enough to enhance your caregiving skills? \\
\hline n (\%) P-values \\
Strongly Disagree & $0(0)$ & \\
Disagree & $9(4.5)$ & $0.000^{* *}$ \\
Agree & $178(89)$ \\
Strongly agree & $13(6.5)$ & \\
\hline
\end{tabular}

${ }^{* *}$ Significant p-values of chi-square test $(\mathrm{P} \leq 0.05)$. Similarly around $89 \%(178)$ of the caregivers stated that communication with the health care professionals is necessary to promote care giving skills (shown in table below).

Table 18. Chi-square test statistic of Q.23.

\begin{tabular}{ccc}
\hline \multicolumn{2}{c}{ Do you think that communication with } \\
professionals is necessary to promote caregiving skills? \\
\hline n (\%) & P-values \\
Strongly Disagree & $0(0)$ & $0.000^{* *}$ \\
Disagree & $9(4.5)$ & \\
Agree & $178(89)$ & \\
Strongly Agree & $13(6.5)$ &
\end{tabular}

** Significant $\mathrm{p}$-values of chi-square test $(\mathrm{P} \leq 0.05)$.

\section{Discussion}

The current study's findings revealed four significant forms of caregiving burdens, including physical, psychological, social, and financial burdens. The mean score for the overall burden of care explicitly indicates that the participants perceived the burden of care, unanimously. The current study concluded that the mean score for the financial burden was the highest whereas; the mean physical burden was calculated to be the lowest. The financial burden could have been the highest in this study due to the low earning capacity of the participants. In addition, the participants did not have any form of rebate or health insurance.

The reason why physical burden was found to be lowest in this study could be due to the fact that the care receivers were independent and were able to perform their activities of daily living by themselves. The demographic variables of the caregivers in our study that were found to be insignificant include relationship to patient, total number of care givers available, marital status, and income. The main reason for this could be the trend of the extended family system, which is a preferred family system in Pakistan. Thus, the extended family system renders a large number of caregivers available at home, reducing the burden of the informal caregiver.

The current study findings also identified professional support as a significant component in reducing the burden of care for the informal caregivers [16]. Professional support to the informal caregivers by health care personnel includes the teaching related to symptom management and the enhancement of care giving 
skills. This helps the caregivers to deal with the problems that may arise after chemotherapy in the forms of side effects. This study is the first of its kind in Pakistan that explores the burden of care among the informal caregivers of patients receiving chemotherapy in a day care setting of a tertiary hospital. This study was conducted on all the cancer patients receiving chemotherapy in the day care, regardless of any specific type and site of cancer. The same study can be replicated on cancer patients with a specified type and site of cancer, so that the caregiver's burden related to a specific type of cancer can be evaluated.

The fact that the Modified Zarit Burden Interview Scale was utilized in this investigation is also strength of this study, as this tool was successfully used for the very first time in Pakistan to explore the physical, financial, psychological, and social burden of informal caregivers. This study can be replicated with similar groups of participants in the public sector in Pakistan. The study was conducted in one single private hospital that provides treatment to patients belonging to different social strata and cultures.

Primarily the study was conducted in one of the private hospitals in Karachi, and the population was targeted through convenient sampling, which led to limited generalizability. Secondly, the cross sectional nature of this study only helped to explore the phenomenon at one point in time, but the long term effects of the phenomenon could be explored by conducting longitudinal studies. Thirdly, some aspects of caregiving, such as caregiving challenges, caregiving preparedness, family function, social support, interventional strategies for the informal caregivers, and positive aspects of caregiving were not assessed in this study. The limited time and resources did not allow taking all these considerations in a single small scale study. Further studies could explore other variables in order to assess the nature of the caregiving burden comprehensively. As the ZBIS is structured and limited, it may have missed some of the caregiving aspects that caregivers might have wanted to share. Therefore, a qualitative research could provide more insight and a deeper understanding of the caregiving burden. Moreover, the study tool can be modified contextually for implementation in further studies in Pakistan.

\section{Conclusion}

Clinical policy can be modified by mandatory face to face health education sessions by health care professionals to enhance the informal caregivers' knowledge regarding symptom management, along with clearing up misconceptions and beliefs related to cancer; distributing written materials based on common caregiving challenges that informal caregivers encounter in the oncology day care center for the caregivers accompanying the patients; providing chemotherapy drugs rebate to reduce the financial burden on the informal caregivers or patients should be referred to NGOs for the reimbursement of the chemotherapy drugs; launching general awareness programs via print and electronic media to promote the caregiving skills. Financial burden was found to be the most preva- 
lent form of burden followed by social, psychological and physical burden respectively. Moderate magnitude of burden superimposed the mild and severe magnitude of burden. Three of the demographic variables including religion, employment status and functional level of care receiver were found to be significant along with some of the variables from ZBIS depicting financial, social, psychological and physical domains.

\section{Acknowledgements}

Authors would like to acknowledge Ms. Mehnaz Hanzala, Ms. Fatima Shahbuddin, Saadia Dawoodani, Asif Ghouri, class fellows, families, and friends for their continued support and feedback.

\section{Conflicts of Interest}

The authors declare no conflicts of interest regarding the publication of this paper.

\section{References}

[1] International Agency for Research on Cancer (2012) World Cancer Fact Sheet. World Health Organization, Geneva, Switzerland.

[2] Anderson, C.S. and Curran, W.J. (2010) Combined Modality Therapy for Stage III Non-Small-Cell Lung Cancer. Seminars in Radiation Oncology, 20, 186-191. https://doi.org/10.1016/j.semradonc.2010.01.007

[3] Giordano, K.F. and Jatoi, A. (2005) The Cancer Anorexia/Weight Loss Syndrome: Therapeutic Challenges. Current Oncology Reports, 7, 271-276. https://doi.org/10.1007/s11912-005-0050-9

[4] Escalante, C.P. and Manzullo, E.F. (2009) Cancer-Related Fatigue: The Approach and Treatment. Journal of General Internal Medicine, 24, 412-416. https://doi.org/10.1007/s11606-009-1056-Z

[5] Chen, J.P., Lo, Y., Yu, C.J., Hsu, C., Shih, J.Y. and Yang, C.H. (2008) Predictors of Toxicity of Weekly Docetaxel in Chemotherapy-Treated Non-Small Cell Lung Cancers. Lung Cancer, 60, 92-97. https://doi.org/10.1016/j.lungcan.2007.09.004

[6] Nijboer, C., Tempelaar, R., Sanderman, R., Triemstra, M., Spruijt, R.J. and Van den Bos, G.A. (1998) Cancer and Caregiving: The Impact on the Caregiver's Health. Psycho-Oncology, 7, 3-13. https://doi.org/10.1002/(SICI)1099-1611(199801/02)7:1<3::AID-PON320>3.0.CO;25

[7] Bauer, J.M. and Sousa-Poza, A. (2015) Impacts of Informal Caregiving on Caregiver Employment, Health, and Family. Journal of Population Ageing, 8, 113-145. https://doi.org/10.1007/s12062-015-9116-0

[8] Kim, Y. and Schulz, R. (2008) Family Caregivers' Strains: Comparative Analysis of Cancer Caregiving with Dementia, Diabetes, and Frail Elderly Caregiving. Journal of Aging and Health, 20, 483-503. https://doi.org/10.1177/0898264308317533

[9] Schubart, J.R., Kinzie, M.B. and Farace, E. (2008) Caring for the Brain Tumor Patient: Family Caregiver Burden and Unmet Needs. Neuro-Oncology, 10, 61-72. https://doi.org/10.1215/15228517-2007-040

[10] Beesley, V.L., Price, M.A. and Webb, P.M. (2011) Loss of Lifestyle: Health Beha- 
viour and Weight Changes after Becoming a Caregiver of a Family Member Diagnosed with Ovarian Cancer. Supportive Care in Cancer, 19, 1949-1956.

http://link.springer.com/article/10.1007/s00520-010-1035-2

https://doi.org/10.1007/s00520-010-1035-2

[11] Kim, Y., Baker, F., Spillers, R.L. and Wellisch, D.K. (2006) Psychological Adjustment of Cancer Caregivers with Multiple Roles. Psycho-Oncology, 15, 795-804.

https://doi.org/10.1002/pon.1013

[12] Cora, A., Partinico, M., Munafo, M. and Palomba, D. (2012) Health Risk Factors in Caregivers of Terminal Cancer Patients: A Pilot Study. Cancer Nursing, 35, 38-47. https://doi.org/10.1097/NCC.0b013e31820d0c23

[13] Segrin, C., Badger, T., Dorros, S.M., Meek, P. and Lopez, A.M. (2007) Interdependent Anxiety and Psychological Distress in Women with Breast Cancer and Their Partners. Psycho-Oncology, 16, 634-643. https://doi.org/10.1002/pon.1111

[14] Kim, Y., Kashy, D.A., Spillers, R.L. and Evans, T.V. (2010) Needs Assessment of Family Caregivers of Cancer Survivors: Three Cohorts' Comparison. Psycho-Oncology, 19, 573-582. https://doi.org/10.1002/pon.1597

[15] Papastavrou, E., Charalambous, A. and Tsangari, H. (2009) Exploring the Other Side of Cancer Care: The Informal Caregiver. European Journal of Oncology Nursing, 13, 128-136. https://doi.org/10.1016/j.ejon.2009.02.003

[16] Belgacem, B., Auclair, C., Fedor, M.C., Brugnon, D., Blanquet, M., Tournilhac, O. and Gerbaud, L. (2013) A Caregiver Educational Program Improves Quality of Life and Burden for Cancer Patients and Their Caregivers: A Randomized Clinical Trial. European Journal of Oncology Nursing, 17, 870-876.

https://doi.org/10.1016/j.ejon.2013.04.006 